\title{
The Human Experience of Crooked Data
}

\author{
CARRIE IDA EDINGER \\ Independent Artist
}

\begin{abstract}
A critical review of Crooked Data: (Mis) Communication in Contemporary Art, an exhibition at the Harnett Museum of Art at the University of Richmond, February 9 - May 5,2017.
\end{abstract}

The conventional paradigm of data has been visually extended by the twenty-one contemporary artists and studios curated in the Crooked Data: (Mis) Communication in Contemporary Art exhibition in the Harnett Museum of Art at the University of Richmond. Data placed within the creative process and data visualizations are the subject matter of this exhibition and work ranges from traditional fine art mediums to new media. Each piece in Crooked Data presents an element of visual curiosity that made me consider the content and the creative process involved from the specific data being presented.

Data is not only being analyzed and utilized as an adaptable tool, but specific artists were placing themselves in social situations and environments that depended on an element of personal engagement to collect their data. While the finished art objects or outcomes from a project are what was being presented within the gallery spaces, the knowledge of how data was considered in the creative process comes from the artist's perspective. Arranged in the form of a website, the Crooked Data online catalog offers this specific knowledge of the creative process with documented interviews from the artists featured in the exhibition.

Elizabeth Schlatter, the curator of Crooked Data and the Deputy Director and Curator of Exhibitions at the University of Richmond Museums, along with Lindsay Hamm, '17, art conservation (interdisciplinary studies) major at the University of Richmond conducted the artist interviews. The interview format is tailored to the individual artist's practice and includes an introduction to the pieces in the exhibition. Each artist has a designated webpage under the web menu category entitled artists. I was able to investigate the artist's work, which piqued my curiosity with the additional information on the scope and connections to how the data was collected and visually interpreted. I view this interactive element as a valuable addition to the exhibition, especially for the viewer to have an opportunity to consider the creative process while visiting the exhibition. Within the exhibition space, computer stations were available for the gallery viewers to read the artist's interviews. 
Two of the computers in the gallery space featured the online gallery that coincides with the Crooked Data exhibition. The online gallery is part of the Digital America website content. Digital America is an online journal that focuses on the American experience of digital art and culture. The journal began with a course in its namesake and continues to grow with the support of the University of Richmond Department of Art and Art History. The online gallery exhibit has work of three artists that explore the misleading nature of digital data. The Founder and Director of Digital America, Dr. Meghan Rosatelli, conducted these individual artist's interviews, along with some of the students that were enrolled in the fall 2016 Digital America course.

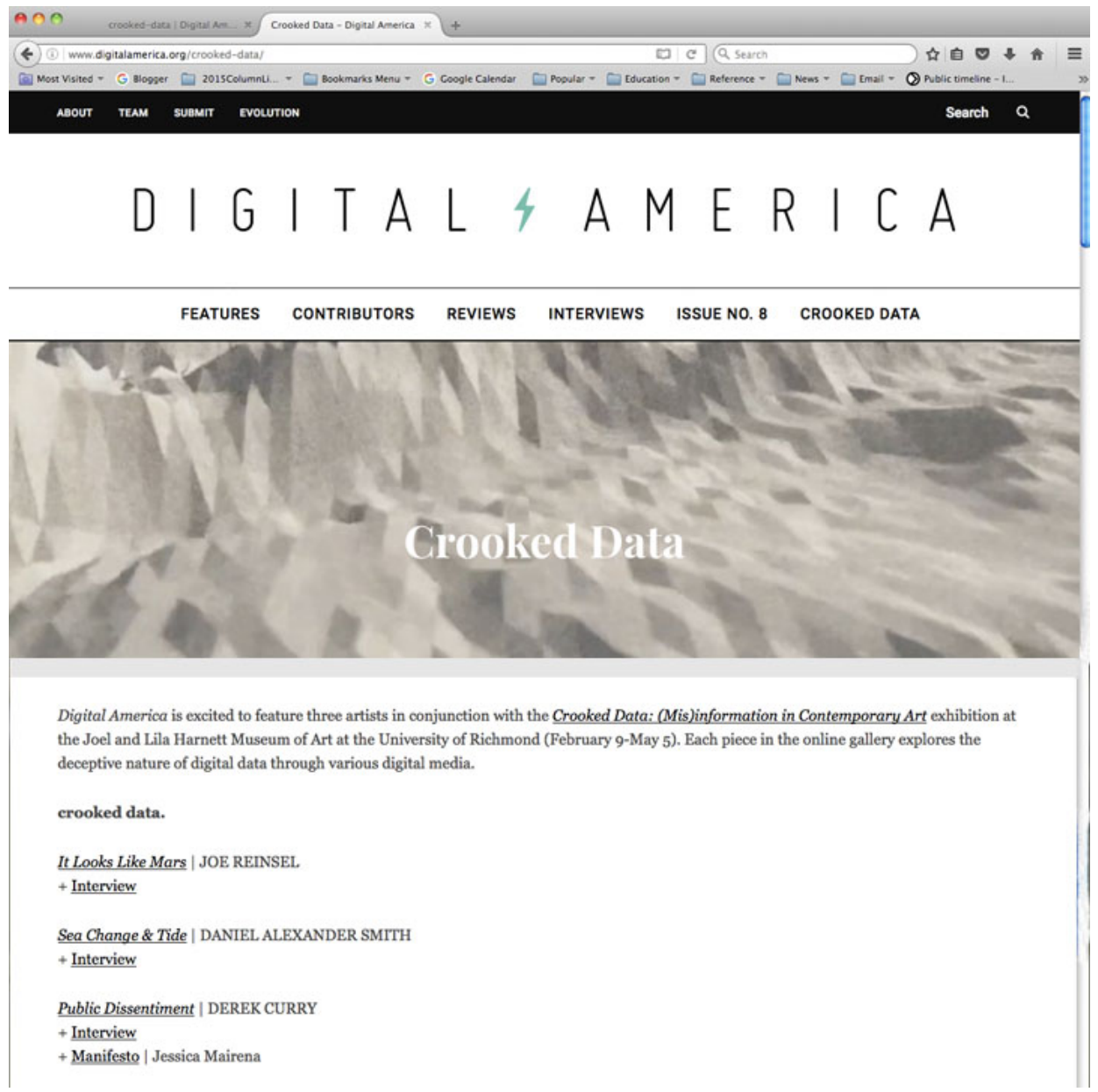

\section{Figure 1. Screenshot of Crooked Data Online Gallery presented on the Digital America Website, 2017 \\ Courtesy of the Digital America Website, University of Richmond Department of Art and Art History}

One of the three online gallery artists, Derek Curry, is investigating online protesting with public engagement and forms of activism via popular social media sites. His academic research and art practice consider the main elements of protest culture, a physical location and a group of people, with the current technological conditions for creating his Public Dissentiment app. Public 
Dissentiment is an algorithmic protest tool that utilizes negative sentiment posts that disrupt the high-frequency trading bots. These trading bots are the analysis tools used to determine the performance of stock trading algorithms. Currently the algorithm process uses an automated decision-making technology that gathers data from algorithmic stock trading and Open Source Intelligence. These algorithms are also scanning to detect uncertainty, which is related to market news and individual social media posts that target businesses. The Public Dissentiment app can be implemented as a direct action protest method at physical site protests or even organized boycotts that employ the everyday interface of the Internet.

Curry's previous research of fake tweets by hacked Twitter accounts is what led his investigations to how trading algorithms perform language processing on the priority of risk aversion over the accuracy of posted information. The history of culture jamming and tactile media is part of the lineage of Public Dissentiment and relates back to the overall theme of the misleading nature of digital data represented in the online gallery. The public opinion posts are meant to utilize cyberspace and intervene with the singular automated decision-making process of the trading bots.

During my time spent on the Public Dissentiment website, I noticed a lot of informative content on the market and protest instructions. I was eager to find out about the engagement with the app during a protest movement. From Curry's interview, he does state that he wants this app to be used in conjunction with physical protests and did not set-up a menu selection with pre-entered names of organizations or corporations that could have influenced people to protest without any real consideration. As the Public Dissentiment project evolves, Curry is open to users contacting him with suggestions for improvement or specific features for their needs.

Relating back to my observation from previewing the exhibition within the physical gallery space, I have chosen two artists who utilize their experiences from the engagement of personal data. R. Luke DuBois investigates data through the contemporary conditions of how we socialize through the engagement of computers in the form of dating sites, while Holly Hanessian explores the sense of touch and brain wave data from the formal behavior of a handshake. Both of my examples explore the momentary elements of personal interactions that have been visually interpreted in a tactile relation. 


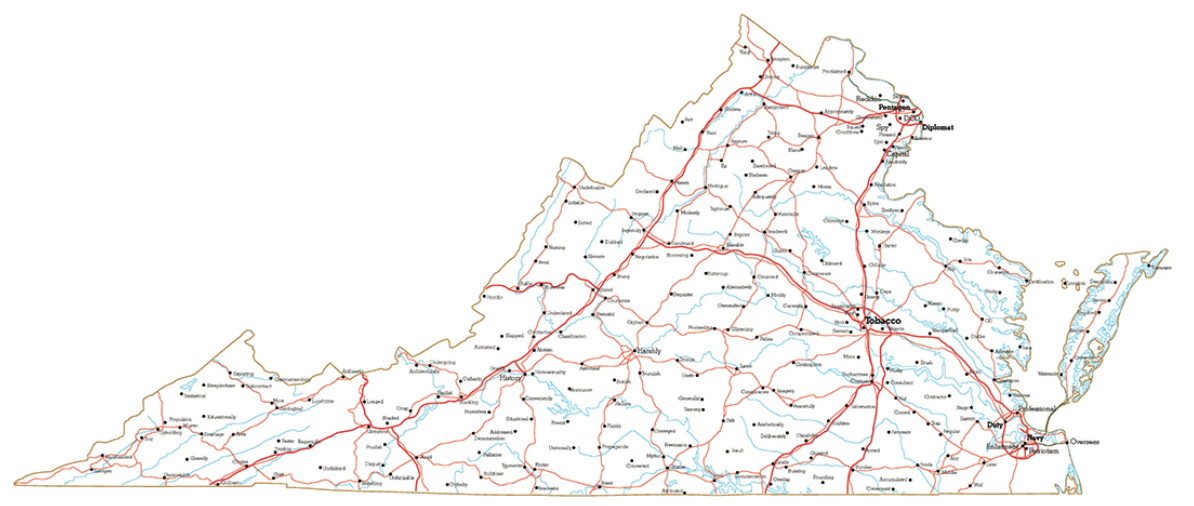

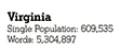

Figure 2. R. Luke DuBois, A More Perfect Union: Virginia, 2011

Pigment-ink on photo rag; $24 \times 36$ inches, Edition of 6 Courtesy of bitforms gallery, New York

R. Luke DuBois' A More Perfect Union series is based on his United States census data obtained from various Internet-based dating sites - single profiles that are presented in a data visualization of a road map. DuBois didn't just download the profiles and sort the personal data from them - he created four types of profiles: a straight man, a gay man, a straight woman and a gay woman. These profiles were for his personal engagement with the different sexual identities on the dating sites. He considered how these profiles were created in each of the United States zip codes on the dating websites, and this became one of the main categories for sorting the data. DuBois algorithmically generated unique words for each specific geographic area of each state. He intentionally generated data that would not fall into established socio-economic statistic categories; instead keywords from the individual profiles have a relation to socio-cultural identities and to concepts of love.

From DuBois' singles population census he has replaced the town and city names with the top generated words from the descriptive words used in the individual biographies. DuBois' gained experience with the Internet-based dating sites by surfing the net himself, but these pigment-ink on photo rag paper data visualizations reference the tangible object of a map that I could identify with the act of physical traveling. For example, as I viewed the map of the State of Virginia, I visually followed my southern route down the Interstate 95 corridor, which I traveled to the University of Richmond Museums. I found Alexandria, Virginia's name replaced with the word Pentagon and read the words replacing smaller populated areas till I got to the word Tobacco that took the place of Richmond, Virginia. The new socio-cultural identities of my travels intrigued me and lead me to the gallery-based computer station to read through DuBois' interview. From the interview DuBois remarks how some of the words are stereotypical, but other data is 
associated with a particular locality. At the end of the interview, DuBois reflects on his beginning assumptions with the project, he assumed that the data he collected would be more related to generic words. Instead the process revealed more of an eye-opener for him to how the singles population identifies themselves.

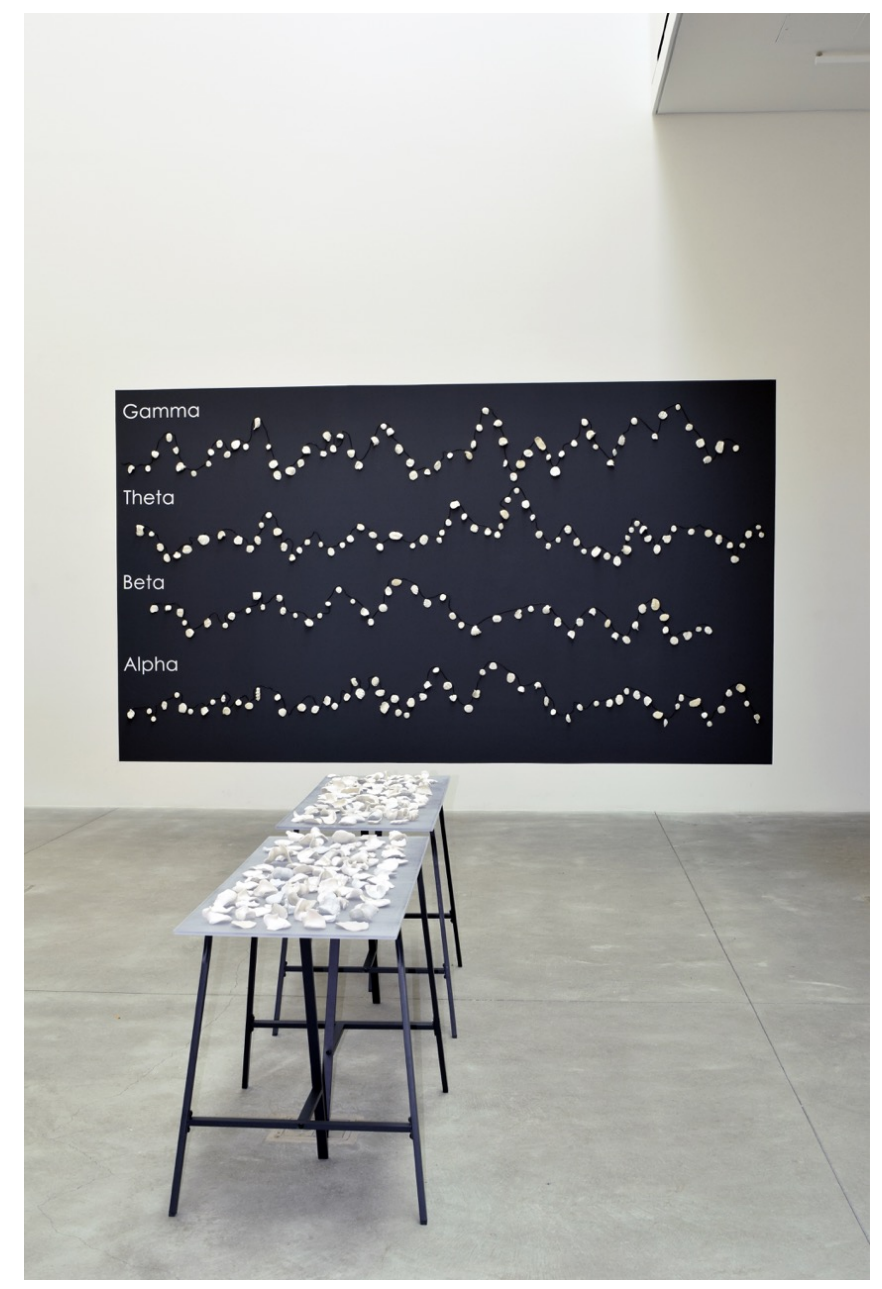

Figure 3. Holly Hanessian, Touch in Real Time, 2014 paint, wire, ceramic hand-prints, vinyl letters; 96 x 168 inches Courtesy of the artist

Holly Hanessian's data visualization for Touch in Real Time is presented as an installation within the gallery exhibition. The data visualization is a reference to the technological tool, electroencephalogram or EEG, which measures and visually reveals electrical patterns from the human brain. Specific tangible materials were used to represent the momentary connections from human engagement. Hanessian's interest in collecting data begins with the common handshake. In her process of collecting data from this human experience she redefined this brief contact into a reflective moment between herself and a participant. 
A handshake is a moment from a type of mutual connection through the interlocking of hands and eye contact. Hanessian utilizes the concept behind the handshake a step further by slowing down this brief act of touch into an intimate interaction for a period of five minutes. The additional allotted time is meant to be a moment of reflection between Hanessian and the participant. The second component concerning the additional time period is the medium, which captures the ephemerality from this specific human experience. Along with documenting individual hand prints, wet clay is pressed and captured in between the negative space of Hanessian's and the participant's hands. The organic shape that the wet clay reveals becomes an artifact from that moment of engagement. The fired porcelain pieces are then arranged within Hanessian's installation.

The data visualization is arranged by referencing the EEG system that records the types of brain waves, Gamma, Beta, Alpha and Theta. This visually depicts specific brain waves from the physical connection of two people touching and being $100 \%$ present with the other person in a handshake. Hanessian had an opportunity to work with neuroscientists to map electrical patterns of the brain and individual bonding hormones that took place during the intimate act of a handshake. From Hanessian's interview on the Crooked Data catalog, she goes into further depth pertaining to the materials and the visual from the EEG depicted in her installation. She refers to the clay artifacts from the handshake as the analogue counterpoint to our current means of digital communication.

The three artists in this exhibition examine a range of personal engagement with data from cyber space to the human body. Whether perceived as a miscommunication or as a connection this specific data's impact within the creative process resonates from the human experience. The overall content from the Crooked Data physical gallery exhibition reaches beyond a standard visual inquiry for the viewer. This exhibition entices the viewer to interact with digital devices that have specific data stored, as well as to ponder the data sources and intent. These heavilylayered creative processes have produced a source of knowledge, while evolving the artist inquiry.

\section{REFERENCES}

http://www/crookeddata.com/

http://www.digitalamerica.org/crookeddata/

http://www.digitalamerica.org/

\section{AUTHOR BIO}

Carrie Ida's projects with interdisciplinary practice investigate cross-discipline approaches and new media. She has published and given research-related presentations with art and anthropological professional organizations. Her public raffle concept was included in the Spring 2016 On the Line program at the University of California, Riverside, and her Collection Project was included in OUT OF CONTEXT, an online exhibition curated by Heit \& Haikes with a limited engagement for Philly Tech Week 2017 in Philadelphia, Pennsylvania. Most recently, 10 Yrs. Later, her sound art piece was included in Digital America's online exhibition that is part of Sound Arts Richmond in Richmond, Virginia. 